\title{
Spinal Cord Ischemia: Practical Imaging Tips, Pearls, and Pitfalls
}

\author{
M.I. Vargas, J. Gariani, R. Sztajzel, I. Barnaure-Nachbar, B.M. Delattre, K.O. Lovblad, and J.-L. Dietemann
}

\section{ABSTRACT}

SUMMARY: Ischemia of the spinal cord is a rare entity with a poor prognosis. Brain ischemia is no longer a diagnostic challenge; on the contrary, ischemia of the spinal cord remains difficult, particularly in children. In this article, we illustrate the principal causes in children and adults, clinical presentation, different techniques for the diagnosis by MR imaging (diffusion, spinal MR angiography, and 1.5 versus 3T), pathophysiology, and differential diagnosis. We will discuss current knowledge, perspectives, and pitfalls.

$l_{k=1}^{\infty}$ schemia of the spinal cord is rare, its prevalence is not wellknown, and it has a poor prognosis. It accounts for approximately $6 \%$ of all acute myelopathies and approximately $1 \%-2 \%$ of all vascular neurologic pathologies. ${ }^{1}$

Several causes are implicated in ischemia of the spinal cord, which differ in the pediatric and adult populations. In children, the most common causes are cardiac malformations and trauma. In adults, atheromatosis is one the principal causes, but thoracoabdominal aneurysms, aortic surgery, ${ }^{2}$ embolic disease, dissection, systemic hypotension, spinal arteriovenous malformations, diving, ${ }^{3}$ coagulopathies, cocaine, ${ }^{4}$ sickle cell disease, ${ }^{5}$ and idiopathic causes are also implicated.

In the past decade, new methods such as diffusion imaging of the spinal $\operatorname{cord}^{6,7}$ allow a better analysis in the acute setting.

In this article, we describe the technical protocols, differential diagnosis, pitfalls, and solutions.

\section{Clinical Presentation}

Clinical presentation depends mainly on the location and extent of the infarction. As with cerebral infarction, the onset of spinal cord infarction is typically abrupt. Most patients develop symptoms quickly, with a maximal symptomatology reached within 12 hours for $>50 \%$ of patients and within 72 hours for most patients. ${ }^{8}$ The neurologic presentation of spinal cord infarction is largely defined by the vascular territory involved. The severity of the impairments can

From the Divisions of Neuroradiology (M.I.V., I.B.-N., K.O.L.), Radiology (J.G., B.M.D.), and Neurology (R.S.), Geneva University Hospitals, Geneva, Switzerland; and Division of Radiology (J.-L.D.), Strasbourg University Hospitals, Strasbourg, France.

Please address correspondence to Maria Isabel Vargas, MD, Geneva University Hospitals, Division of Neuroradiology, DISIM, Rue Gabrielle-Perret-Gentil 4, 1211 Genève 14, Switzerland; e-mail: Maria.I.Vargas@hcuge.ch

- Indicates open access to non-subscribers at www.ajnr.org

http://dx.doi.org/10.3174/ajnr.A4118 vary widely, from paraplegia to minor weakness. The involved cord level can be anywhere along the length of the cord. Back pain often accompanies spinal cord ischemia and has been reported in as many as $70 \%$ of patients, typically at the level of the lesion. ${ }^{8}$

\section{Different Clinical Manifestations}

Anterior Spinal Artery Syndrome. The most common clinical presentation of a spinal cord infarction is anterior spinal artery syndrome. Anterior spinal artery infarct typically presents as a bilateral loss of motor function and pain/temperature sensation, with relative sparing of proprioception and vibratory senses below the level of the lesion. The acute stages are characterized by flaccidity and loss of deep tendon reflexes; spasticity and hyperreflexia develop during ensuing days and weeks. Autonomic dysfunction may be present and can manifest as hypotension (either orthostatic or frank hypotension), sexual dysfunction, and/or bowel and bladder dysfunction. If the lesion is in the rostral cervical cord, respiration is compromised.

Incomplete Syndrome of the Spinal Artery Syndrome. Ischemia may be localized at the level of the anterior horns; in this case, clinical presentations may be the following:

1) Acute paraplegia (pseudopoliomyelitic form) without sensory abnormalities and without sphincter dysfunction

2) Painful bilateral brachial diplegia in the case of a cervical lesion ${\text { (the man-in-the-barrel syndrome })^{9}}^{9}$

3) Progressive distal amyotrophy due to chronic lesions of the anterior horns; this form may be misdiagnosed as lateral amyotrophic sclerosis.

Posterior Spinal Artery Syndrome. Posterior spinal artery infarction produces loss of proprioception and vibratory senses below the level of the injury and total anesthesia at the level of the injury. 
Weakness has been described but is typically mild and transient. Unilateral involvement is more common, but bilateral presentations have also been described. ${ }^{8}$

\section{Other Less Typical Presentations.}

1) Sulcocommissural syndrome presents as a partial Brown-Sequard syndrome clinical picture with sparing of postural sen-

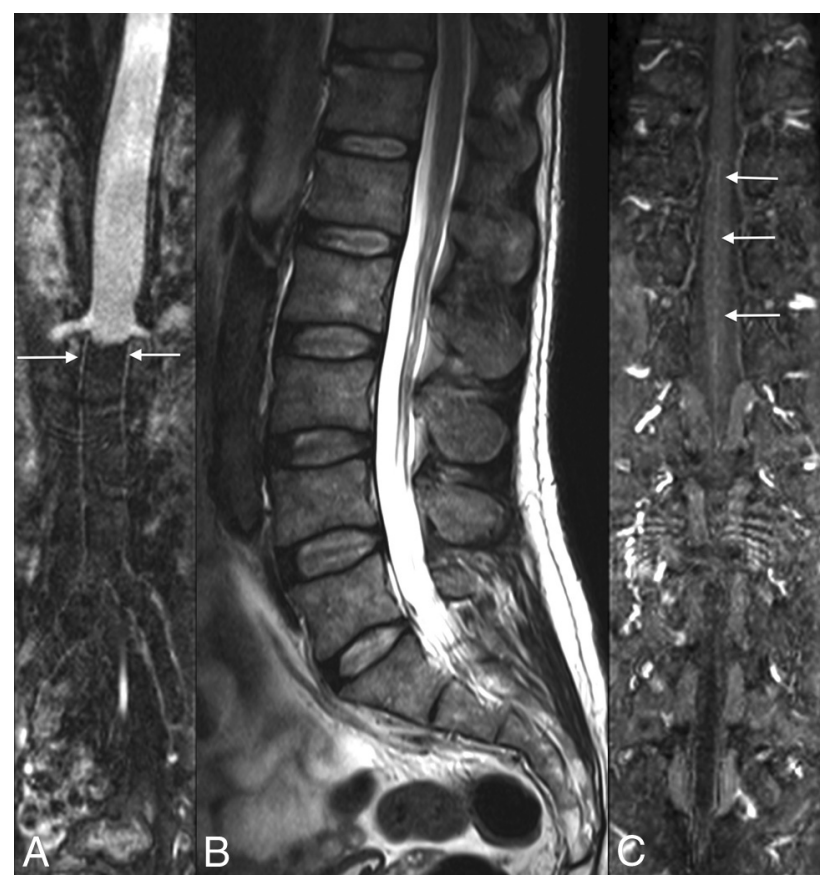

FIG 1. Localization of the artery of Adamkiewicz in a patient with aortic thrombus. MR angiography shows the thrombus in the abdominal aorta below the renal arteries (arrows, $A$ ). No ischemia is visible in the conus medullaris $(B)$. The artery of Adamkiewicz is permeable (arrows, C). sibility. The syndrome consists of hemiparesis with a contralateral spinothalamic sensory deficit.

2) Infarction at the level of conus medullaris may be misdiagnosed as a cauda equina syndrome.

3) Central spinal infarct occurs after cardiac arrest or prolonged hypotension; its clinical presentation includes bilateral spinothalamic sensory deficit with sparing of the posterior columns. Motor deficit and sphincter dysfunction are usually absent.

4) Transverse medullary infarction (full transverse lesions) presents with sudden and severe inability to walk due to paraplegia or paraparesis or, in cases of higher cord lesions, tetraplegia or tetraparesis. There may be complete sensory loss involving all modalities. Pain is reported as tightness radiating circumferentially. Sphincter dysfunction is present with loss of bladder and bowel control. It is often of embolic origin.

Spinal Transient Ischemic Attacks. Transient symptoms lasting a few minutes to several hours, so-called spinal transient ischemic attacks, have also been described in a variety of clinical settings, but these are unusual. ${ }^{8}$

\section{Vascularization of the Spinal Cord}

The vascularization of the spinal cord is supplied principally by the anterior spinal artery, $0.2-0.8 \mathrm{~mm}$; posterolateral spinal artery, $0.1-0.4 \mathrm{~mm}$; and arteria radicularis magna or artery of Adamkiewicz, $0.5-1.2 \mathrm{~mm}$.

The anterior spinal artery supplies the anterior two-thirds of the spinal cord, situated in the pia mater along the anterior median fissure and descending vertically. It is formed by 2 small branches arising from the fourth segment of the vertebral arteries with fusion at the level of the foramen magnum and branches arising from the vertebral artery, the ascending cervical artery, the inferior thyroid artery, the intercostal arteries, the lumbar artery,

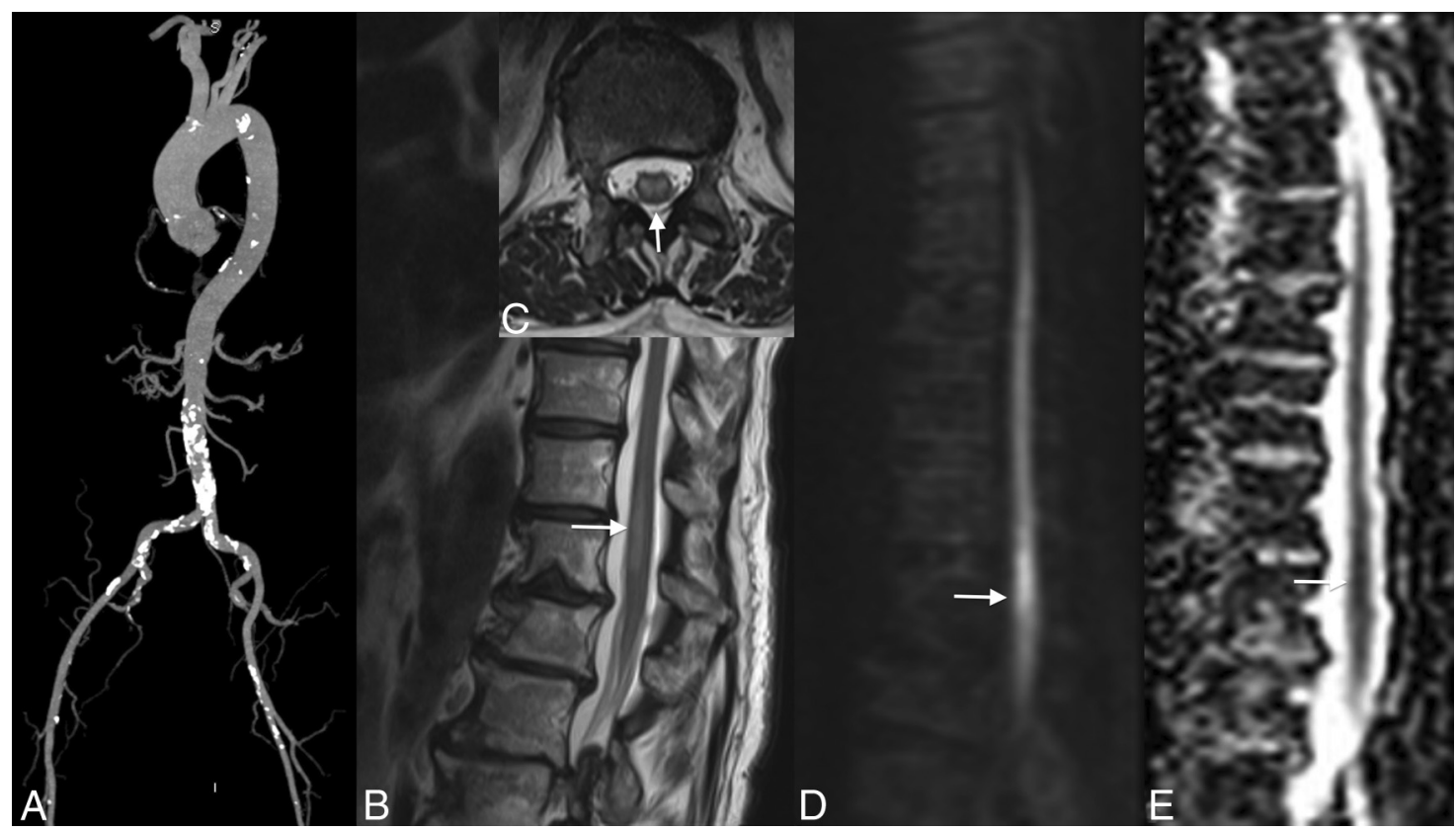

FIG 2. Ischemia provoked by an atheroma. Note the important atheromatosis of the abdominal aorta nicely shown by the volume-rendering reconstruction of $C T$ angiography $(A)$. Ischemia of the conus medullaris shown by MR imaging is hyperintense on $\mathrm{T} 2$ with a restriction of diffusion (arrows, B-E). 


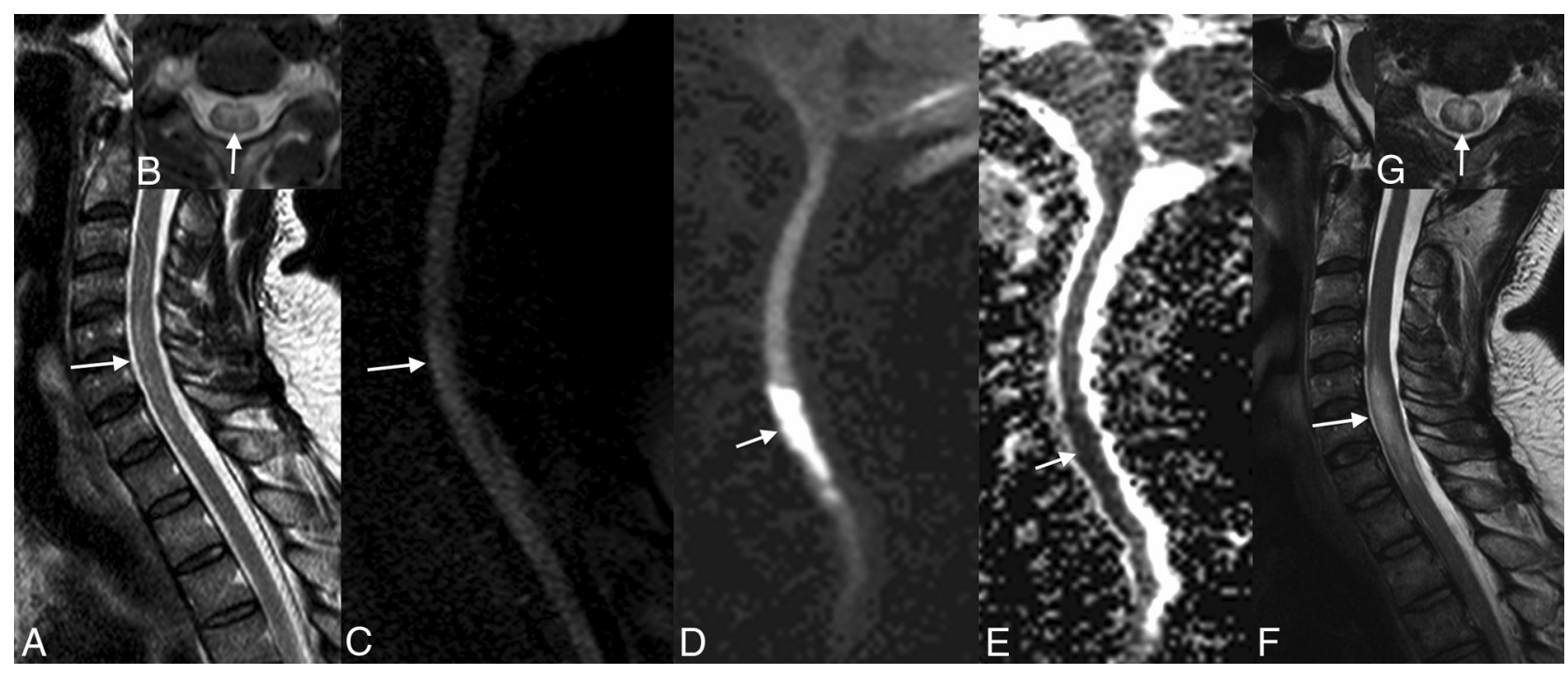

FIG 3. Evolution of ischemia. The first MR image shows the subtle signal anomaly on $\mathrm{T} 2$ and diffusion sequences (arrows, $A-C$ ). Follow-up 48 hours later shows an important tumefaction and high signal on $\mathrm{T} 2 \mathrm{Wl}$ associated with a restriction of diffusion of the cervical spinal cord at the C4-C7 levels (arrows, D-G).

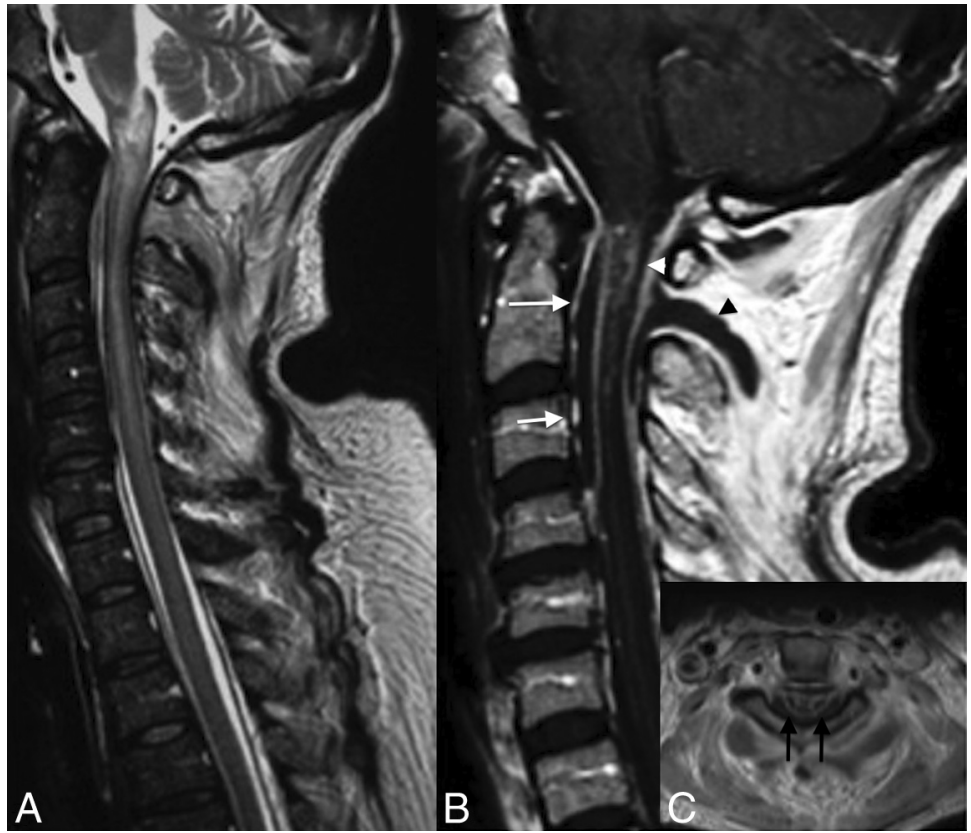

FIG 4. Venous infarction in a patient with epidural and paraspinal abscesses. Note large intramedullary high signal on T2 of the cervical spinal cord $(A)$. TIWI with contrast medium demonstrates an intramedullary enhancement $(B$ and $C$ ), the anterior (arrows, $B$ ) and posterior epidural (white arrowhead, B), and paraspinal abscesses (black arrowhead, $B$ ). Note enhancement on axial $\mathrm{Tl}$ of both sides of the median line, reflecting venous ischemia.

the iliolumbar artery and lateral sacral arteries, and principally by the artery of Adamkiewicz.

The artery of Adamkiewicz or the arteria radicularis magna has a particular "hairpin turn" form and must be differentiated from the anterior radiculomedullary vein, which has the same form but is larger and more tortuous. ${ }^{10}$ It arises on the left side of the aorta between the T8 and L1 segments, to anastomose with the anterior spinal artery and supply the lower two-thirds of the spinal cord (conus medullaris). Lesions of this artery produce motor deficits of the legs and fecal and urinary incontinence.

The 2 posterolateral spinal arteries arise from the posteroinferior cerebellar arteries and supply the posterior third (posterior columns, posterior roots, and dorsal horns) of the spinal cord.

\section{Venous System of the Spinal Cord}

The venous system is divided into intrinsic and extrinsic systems. The intrinsic veins are divided into sulcal and radial veins, and the extrinsic veins are composed of the anterior and posterior spinal veins. The anterior median spinal vein ${ }^{11}$ runs with the anterior spinal artery and continues to the filum terminale vein. One posterior median, the greatest spinal vein, is accompanied by 2 posterolateral veins.

The extrinsic system is in contact with the spinal pia matter and includes the pial venous network, the longitudinal collectors, and the radicular veins. This configuration produces large lateral and dorsoventral anastomotic systems. ${ }^{12}$

Spinal veins drain into the anterior and posterior radiculomedullary veins, which in turn drain into the paravertebral and intervertebral plexuses. $^{13}$ These venous plexuses drain into the segmental veins, draining into the ascending lumbar veins, azygos system, and pelvic venous plexuses.

The radiculomedullary veins communicate with the epidural venous system. There are 3 levels of intercommunicating veins ${ }^{14}$ :

1) The internal vertebral venous plexus, formed by intradural and epidural veins, communicating with the intracranial

AJNR Am J Neuroradiol 36:825-30 May 2015 www.ajnr.org 


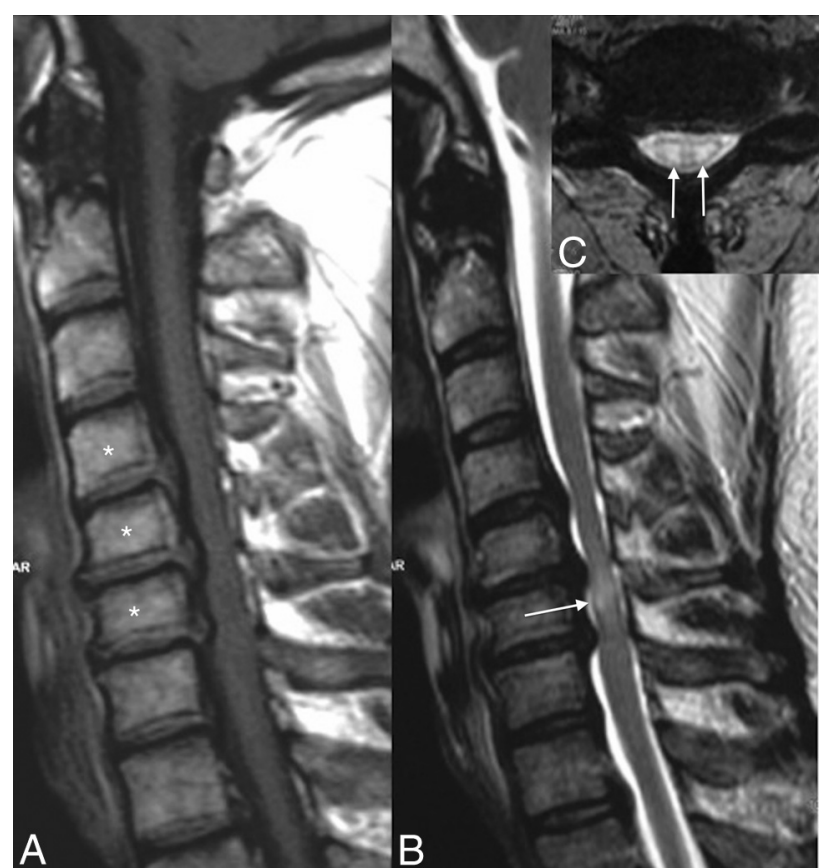

FIG 5. Cervical spinal canal stenosis and venous infarction. Note the cervical spinal canal stenosis from C4 to C6 due to cervical spondylosis (asterisks, $A$ ) and the intramedullary high signal on T2WI (arrow, $B$ ) at the same level with the "snake eye" appearance on axial T2WI (arrows, C).

venous system and draining into the external vertebral veins

2) The external vertebral venous plexus, located around the vertebra

3) The basivertebral veins, within the vertebra.

\section{Pathophysiologic Mechanisms}

The origin of ischemia in adults is primarily an embolus (Fig 1) or plaque (Fig 2) that leads to the occlusion of an artery. When systemic hypotension is the cause, the mechanism is the same as that in ischemia resulting from an overdose of $\beta$ blockers (Fig 3), with the lesion appearing in the watershed areas.

Other sources are also implicated such as the following:

- Cardiac surgery and minimally invasive procedures

- Compression of the radicular artery by a disk ${ }^{15,16}$

- Cervical degenerative canal associated with minor trauma.

Venous origin is also involved in cases of the following:

1) Arteriovenous fistulas leading to an increased venous pressure with, first, a vasogenic edema appearing as a hyperintensity on $\mathrm{T} 2$, which may enhance. This persistence can lead to ischemia if the malformation is not treated and can mimic a subacute arterial ischemic lesion

2) Coagulopathies

3) Epidural infection leading to epidural venous thrombosis with secondary spinal cord infarction (Fig 4)

4) Myelopathy related to cervical stenosis, in most cases, related to chronic venous infarction responsible for the classic "snakeeye" sign (Fig 5).

Last, anatomy can play an important role in the origin of ischemia as illustrated by Gailloud et al. ${ }^{17}$ Their article shows proxi-
Table 1: Technical MR imaging parameters of the spinal cord protocol at 3T

\begin{tabular}{lrrcc}
\hline Sequences & TR $(\mathbf{m s})$ & TE $(\mathbf{m s})$ & $\begin{array}{c}\text { Section } \\
\text { Thickness }(\mathbf{m m})\end{array}$ & B0 \\
\hline SE T1 & 670 & 10 & 3 & \\
SE T2 & 4000 & 128 & 3 & \\
STIR & 5860 & 108 & 3 & \\
Axial GE T2 & 450 & 17 & 3 & \\
Axial SE T2 & 4000 & 124 & 3 & $b=500-700$ \\
Diffusion & 2600 & 68 & 3 & $b=500-800$, \\
DTI & 2600 & 73 & 2 & $20-25$ directions \\
& & & &
\end{tabular}

Note:-SE indicates spin-echo; GE, gradient-echo.

Table 2: Technical MR imaging parameters of the spinal cord protocol at 1.5T

\begin{tabular}{lrrcc}
\hline Sequences & TR $(\mathbf{m s})$ & TE $(\mathbf{m s})$ & $\begin{array}{c}\text { Section } \\
\text { Thickness }(\mathbf{m m})\end{array}$ & B0 \\
\hline SE T1 & 590 & 10 & 3 & \\
SE T2 & 3270 & 71 & 3 & \\
STIR & 3000 & 38 & 3 & \\
Axial GE T2 & 590 & 24 & 3 & \\
Axial SE T2 & 4640 & 79 & 3 & $b=500-700$ \\
Diffusion & 6000 & 67 & 3 & $b=500-800$, \\
DTI & 3200 & 67 & 3 & $20-25$ directions \\
& & & &
\end{tabular}

Note:-SE indicates spin-echo; GE, gradient-echo.

mal non-ostial intersegmental artery stenosis at the upper thoracic level, resulting from the leftward deviation of the descending aorta and the existence of a fixed point along the course of the intersegmental arteries.

In children, minor trauma is a cause of ischemia related to fibrocartilage emboli ${ }^{18}$ and also spasm. Other origins include complications of cardiac surgery or traction for scoliosis after orthopedic surgery, ${ }^{19}$ sickle cell anemia, and umbilical artery catheter in the neonate. Few series in the literature exist concerning ischemia in children. ${ }^{20,21}$ Stettler et $\mathrm{al}^{20}$ found that the most affected territory is the spinal anterior artery. The differential diagnosis includes idiopathic myelopathy and infectious and inflammatory myelitis.

\section{Imaging}

Diffusion revolutionized the diagnosis of brain ischemia in the early 90s; however, it has only been used for the spine in the past decade $e^{6,22}$ and remains a technical challenge because of the need for strong gradients, the size of the spinal cord, flow artifacts, and so forth. A few series in the literature concern the use of this technique in ischemia. ${ }^{22,23} \mathrm{CT}$ and conventional angiography are not useful for the diagnosis; however in older patients, the visualization of atheromatosis or vascular lesions (aorta, lumbar arteries) after surgery can orient the diagnosis.

MR imaging is the examination of choice for the diagnosis of ischemia and for the differential diagnosis; the following technical protocol is recommended at $1.5 \mathrm{~T}$ and $3 \mathrm{~T}$ (see Tables 1 and 2 for details of parameters): sagittal spin-echo T2 or T2 STIR, sagittal spin-echo T1, axial gradient-echo T2 at the cervical and dorsal levels, spin-echo T2 at the medullary conus, and diffusion-weighted images in the sagittal or axial planes. The advantage of the sagittal plane is the larger coverage with a shorter acquisition time. However, the axial plane is useful to 


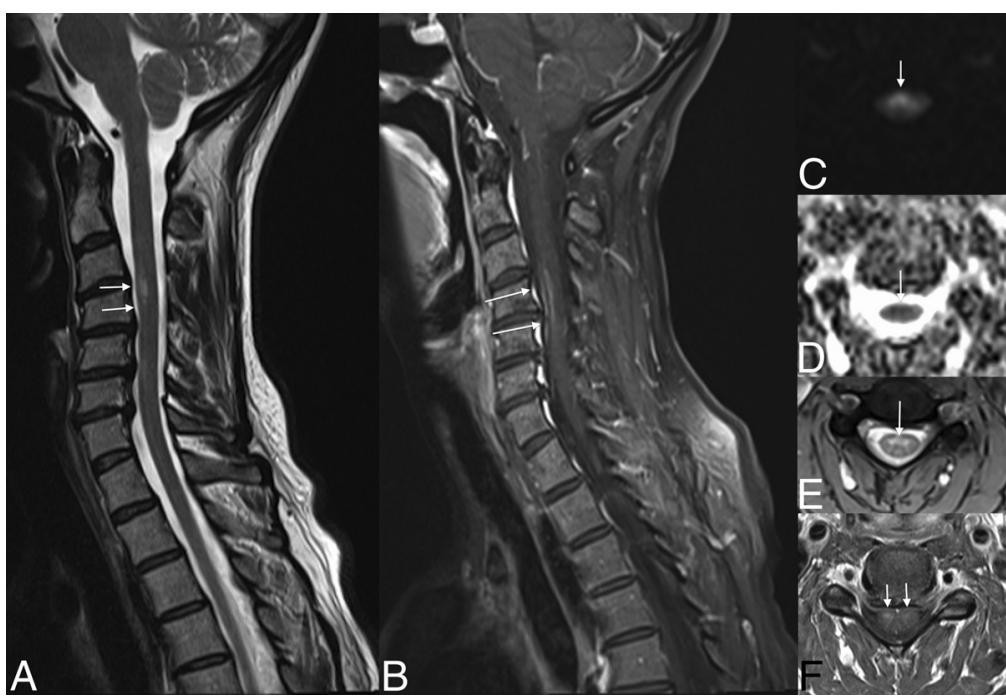

FIG 6. Subacute ischemia. Note the slight hypersignal of the spinal anterior territory at the level of C4-C6 on T2WI (arrows, $A$ and E), associated with a restriction of diffusion (arrows, $C$ and D) and enhancement (arrows, $D$ ).

visualize hypersignal on both sides of the median line in cases of ischemia of the spinal anterior artery. ${ }^{23,24}$ This axial plane can be useful to differentiate ischemia from other entities. DTI is primarily used in research, and a few centers use it in clinical practice. Its contribution in this disease is minor.

Contrast media can aid in the diagnosis in the acute stage (Fig 3) because enhancement is absent at this stage and can differentiate it from inflammatory, tumoral, or infectious diseases. The choice of $1.5 \mathrm{~T}$ and $3 \mathrm{~T}$ is controversial for the visualization of ischemia. Imaging of the cervical spine is of higher quality at 3T; however, artifacts remain at the thoracic level. ${ }^{25}$ In clinical routine, an adaptation of the FOV and resolution of MR images in children by age is recommended.

In case of suspicion of a thrombus in the abdominal aorta, the visualization of the artery of Adamkiewicz by MR angiography can help in the diagnosis.

\section{Patterns of Ischemia of the Spinal Cord}

In the acute stage, ischemia presents as a restriction in diffusionweighted imaging of the spinal cord, hyperintense signal on T2 and STIR, and isointense on T1 and may be associated with a slight enlargement of the cord, without enhancement, which appears in the subacute phase (Figs 3 and 6).

Depending on the affected artery, the territory touching the white or gray matter will vary and the shape will be different.

Kumral et al ${ }^{26}$ and Novy et al ${ }^{8}$ illustrated the different patterns of spinal cord ischemia; the anterior spinal artery territory is limited to the anterior horns bilaterally or unilaterally and the adjacent white matter. The posterior spinal artery infarct is limited to the posterior columns alone or to the surrounding white matter and can be unilateral or bilateral. In the chronic stages, localized atrophy is possible.

In adults, infarction of the vertebral body has been associated with spinal cord ischemia ${ }^{8,27,28}$ associated with high signal and enhancement of the adjacent disk, ${ }^{29}$ which can be explained by the common vascularization of the vertebral body, disk, and spinal cord. Hemorrhagic transformation may occur.

\section{Key Points}

Diffusion imaging is recommended in all acute myelopathies of the spinal cord. The analysis of vessels, such as the aorta and lumbar arteries, can guide the diagnosis. In the acute stage, there is no enhancement in ischemia, which is generally present in inflammatory, tumoral, and infectious pathologies. Major involvement (hyperintensity on T2) of the spinal cord in an acute epidural infection can be related to venous infarcts.

Disclosures: Bénédicte $M$. Delattre-UNRELATED: Employment: Philips Healthcare, ${ }^{*}$ Comments: My institution receives part of my salary from Philips Healthcare; Other: Philips Healthcare, Comments: employee of Philips Healthcare from April 2012 to October 2013. *Money paid to the institution.

\section{REFERENCES}

1. Sandson TA, Friedman JH. Spinal cord infarction: report of $\mathbf{8}$ cases and review of the literature. Medicine 1989;68:282-92

2. Piffaretti G, Bonardelli S, Bellosta R, et al. Spinal cord ischemia after simultaneous and sequential treatment of multilevel aortic disease. J Thorac Cardiovasc Surg 2014;148:1435-42.e1

3. Kamtchum Tatuene J, Pignel R, Pollak P, et al. Neuroimaging of diving-related decompression illness: current knowledge and perspectives. AJNR Am J Neuroradiol 2014;35:2039-44

4. Gorelik N, Tampieri D. Cocaine-induced vasospasm causing spinal cord transient ischemia. Neuroradiol J 2012;25:364-67

5. Márquez JC, Granados AM, Castillo M. MRI of cervical spinal cord infarction in a patient with sickle cell disease. Clin Imaging 2012;36:595-98

6. Vargas MI, Delavelle J, Jlassi H, et al. Clinical applications of diffusion tensor tractography of the spinal cord. Neuroradiology 2008;50:25-29

7. Nogueira RG, Ferreira R, Grant PE, et al. Restricted diffusion in spinal cord infarction demonstrated by magnetic resonance line scan diffusion imaging. Stroke 2012;43:532-35

8. Novy J, Carruzzo A, Maeder P, et al. Spinal cord ischemia: clinical and imaging patterns, pathogenesis, and outcomes in 27 patients. Arch Neurol 2006;63:1113-20

9. Berg D, Mullges W, Koltzenburg M, et al. Man-in-the-barrel syndrome caused by cervical spinal cord infarction. Acta Neurol Scand 1998;97:417-19

10. Bowen BC, DePrima S, Pattany PM, et al. MR angiography of normal intradural vessels of the thoracolumbar spine. AJNR Am J Neuroradiol 1996;17:483-94

11. Thron AK. Vascular anatomy of the spine and spinal cord. In: Hurst RW, Rosenwasser RH, eds. Interventional Neuroradiology. New York: Informa Healthcare; 2008:39-55

12. Moes $P$, Maillot C. Superficial veins of the human spinal cord: an attempt at classification [in French]. Arch Anat Histol Embryol 1981;64:5-110

13. Lasjaunias PL. Functional vascular anatomy of the brain, spinal cord and spine. In: Lasjaunias PL, ed. Surgical Neuroangiography. Vol. 3. New York: Springer-Verlag; 1990

14. Groen RJ, du Toit DF, Phillips FM, et al. Anatomical and pathological considerations in percutaneous vertebroplasty and kyphoplasty: a reappraisal of the vertebral venous system. Spine 2004;29: 1465-71 
15. Hirsch E, Vautravers P, Dietemann JL, et al. Acute lumbar spinal cord disease caused by lumbar disk hernia [in French]. Presse Med 1986;15:843-44

16. Pau A, Cossu M, Turtas S, et al. Spinal cord dysfunction from lumbar disk herniation. Acta Neurol (Napoli) 1989;11:439-43

17. Gailloud P, Ponti A, Gregg L, et al. Focal compression of the upper left thoracic intersegmental arteries as a potential cause of spinal cord ischemia. AJNR Am J Neuroradiol 2014;35:1226-31

18. Reisner A, Gary MF, Chern JJ, et al. Spinal cord infarction following minor trauma in children: fibrocartilaginous embolism as a putative cause. J Neurosurg Pediatr 2013;11:445-50

19. Lewis SJ, Gray R, Holmes LM, et al. Neurophysiological changes in deformity correction of adolescent idiopathic scoliosis with intraoperative skull-femoral traction. Spine 2011;36: 1627-38

20. Stettler S, El-Koussy M, Ritter B, et al. Non-traumatic spinal cord ischaemia in childhood: clinical manifestation, neuroimaging and outcome. Eur J Paediatr Neurol 2013;17:176-84

21. Blennow G, Starck L. Anterior spinal artery syndrome: report of seven cases in childhood. Pediatr Neurosci 1987;13:32-37
22. Thurnher MM, Bammer R. Diffusion-weighted MR imaging (DWI) in spinal cord ischemia. Neuroradiology 2006;48:795-801

23. Loher TJ, Bassetti CL, Lovblad KO, et al. Diffusion-weighted MRI in acute spinal cord ischaemia. Neuroradiology 2003;45:557-61

24. Stepper F, Lovblad KO. Anterior spinal artery stroke demonstrated by echo-planar DWI. Eur Radiol 2001;11:2607-10

25. Vargas MI, Delavelle J, Kohler R, et al. Brain and spine MRI artifacts at 3 Tesla. J Neuroradiol 2009;36:74-81

26. Kumral E, Polat F, Gulluoglu H, et al. Spinal ischaemic stroke: clinical and radiological findings and short-term outcome. Eur J Neurol 2011;18:232-39

27. Yuh WT, Marsh EE 3rd, Wang AK, et al. MR imaging of spinal cord and vertebral body infarction. AJNR Am J Neuroradiol 1992; 13:145-54

28. Faig J, Busse O, Salbeck R. Vertebral body infarction as a confirmatory sign of spinal cord ischemic stroke: report of three cases and review of the literature. Stroke 1998;29:239-43

29. Amoiridis G, Ameridou I, Mavridis M. Intervertebral disk and vertebral body infarction as a confirmatory sign of spinal cord ischemia. Neurology 2004;63:1755 